\title{
Aplicación de técnicas de clustering para caracterizar proveedores de servicios de mantenimiento
}

\section{Applying the clustering technique for characterising maintenance outsourcing}

\author{
Antonio M. Cruz ${ }^{1}$, Sandra P. Usaquén-Perilla ${ }^{1}$, Nidia N. Vanegas-Pabón²
}

y Carolina Lopera ${ }^{1}$

1 Dpto. de Ciencias Básicas, Facultad de Medicina, Universidad del Rosario. Bogotá, Colombia. sandrausaquen@gmail.com, antonio.cruz43@urosario.edu.co, clopera@gmail.com

2 Dpto. de Equipos Médicos, Hospital Universitario de la Samaritana. Bogotá, Colombia. Nidia.vanegas@hus.org.co

Recibido 21 Agosto 2009/Enviado para Modificación 16 Abril 2010/Aceptado 25 Mayo 2010

\section{RESUMEN}

Objetivo Utilizar técnicas de clustering para caracterizar a los proveedores de servicios de mantenimiento de una institución de salud.

Métodos El estudio analiza el inventario del equipamiento perteneciente a 7 áreas pilotos (264 equipos médicos). Se aplican técnicas de clustering usando 26 variables. Entre las más significativas: el tiempo de respuesta (RT), la duración de las intervenciones (DR), la disponibilidad y el tiempo de cambio de estado (TAT). Resultados La obsolescencia del equipo biomédico en promedio es de 0,78 . Se identifican 4 grupos de proveedores de servicios. Grupos (1 y 3): Mejor desempeño, menores valores de TAT, RT y DR; cuyos proveedores son: O, L, C, B, I, S, H, F, G; representan el $56 \%$ del total; con valores de TAT entre: 1,4 días $<$ TAT $<2,56$ días como promedio. Grupo (0): Desempeño medio, cuyos proveedores son: V, M, K, Z, T, Y; representan el $38 \%$; con valores de TAT iguales a 9,79 días como promedio. Grupo (2): con bajos desempeños, por poseer valores altos de TAT (101 días como promedio); con único proveedor: J, representa el $6 \%$ del total de la muestra analizada.

Conclusiones La metodología empleada permitió caracterizar el inventario de equipos médicos y a los proveedores de servicios de mantenimiento. La técnica de conglomerados demostró ser efectiva para identificar los proveedores más competitivos.

Palabras Clave: Disponibilidad de servicios de salud, servicios contratados, análisis por conglomerados, servicio de mantenimiento e ingeniería en hospital, ingeniería biomédica (fuente: DeCS, BIREME).

\section{ABSTRACT}

Objective Using clustering techniques for characterising companies providing health institutions with maintenance services. 
Methods The study analysed seven pilot areas' equipment inventory (264 medical devices). Clustering techniques were applied using 26 variables. Response time (RT), operation duration (OD), availability and turnaround time (TAT) were amongst the most significant ones.

Results Average biomedical equipment obsolescence value was 0.78 . Four service provider clusters were identified: clusters 1 and 3 had better performance, lower TAT, RT and DR values (56\% of the providers coded O, L, C, B, I, S, H, F and $\mathrm{G}$, had 1 to 4 day TAT values: <TAT $<2.56$ days on average). Cluster 0 had medium performance ( $38 \%$ of providers coded $\mathrm{V}, \mathrm{M}, \mathrm{K}, \mathrm{Z}, \mathrm{T}$ and $\mathrm{Y}$, having an average 9.79 TAT value). Cluster 2 ( $6 \%$ - provider $\mathrm{J}$ ) had low performance, having very a high TAT level (101 days on average).

Conclusions The methodology allowed medical equipment inventory and maintenance service suppliers to be characterised. The cluster technique was effective in identifying the most competitive suppliers.

Key Words: Health service accessibility, contract service, cluster analysis, maintenance and engineering, hospital, biomedical engineering (source: MeSH, NLM).

$\mathrm{E}$ 1 ingeniero clínico es un ingeniero biomédico que desde su ámbito, contribuye a que el paciente tenga un entorno seguro (1). Una de las funciones del ingeniero clínico es acompañar y controlar los servicios de mantenimiento que son ejecutados por las empresas externas (tercerización u "Outsourcing") (2). El Outsourcing se puede definir "...como una estrategia empresarial por medio de la cual una organización delega formalmente funciones misionales y no misionales a otra empresa..." $(3,4)$.

La utilización de la tercerización se ha convertido en una práctica muy utilizada en el sector salud. Por ejemplo, términos de volúmenes monetarios; se ha reportado que el monto de dinero destinado a pagar los servicios de los contratos de mantenimiento está en el orden del $64 \%$ del presupuesto destinado a la gestión de tecnologías $(5,6)$. Esta tendencia es debido a que para muchas instituciones de salud muchas veces no posee el recurso humano debidamente entrenado, ni los materiales para realizar estas actividades. Por eso, el desarrollo de un conjunto de herramientas que permitan supervisar el nivel de calidad de los proveedores de servicios de mantenimiento es un factor primordial (7). Ya existen algunas experiencias reportadas por ejemplo, la aplicación de estas herramientas ha contribuido a reducir los gastos que implican los contratos de servicios de mantenimiento hasta en un $20 \%$ (8).

Sin embargo, para los países en desarrollo el problema es aún mayor, pues no se posee suficiente información para realizar estos estudios de evaluación del desempeño. Por ejemplo, un estudio reportado concluyó que en "... en 
Colombia no se han realizado "profundos" estudios sobre este tema, las únicas indagaciones que se han hecho se refieren en alguna medida a la importancia que esta estrategia tiene entre las Instituciones Prestadoras de Servicios de Salud en Colombia...." (3).

Entonces, el problema científico existente, es que aún no ha sido abordado con suficiente profundidad el tema de la evaluación del desempeño de los proveedores de servicios de mantenimiento. El propio Instituto para la Investigación de los Cuidados de Emergencia (por sus siglas en inglés ECRI) ha planteado que a pesar de conocerse cuáles son las variables que caracterizan a los proveedores de servicios de mantenimiento y las variables que miden su desempeño "... aún no existe una formulación universal que permita establecer la calidad del servicio de los proveedores..." (8)

Por eso, el gran aporte a hacer en este campo, es obtener un indicador que permita medir y predecir el desempeño de los proveedores de servicios de mantenimiento en función de las características de las propias instituciones. Pero para obtener un indicador, por una parte, primero hay que caracterizar el inventario de equipos bajo contrato y luego, por otra parte, caracterizar a las instituciones de servicios de mantenimiento responsables de estos equipos; y finalmente, con esta información se podría medir y predecir el desempeño mediante la construcción de un indicador o predictor numérico.

De esta manera el trabajo que aquí se publica es un primer resultado, como paso inicial de un objetivo más abarcador, que es la construcción de un predictor numérico para evaluar el desempeño de los proveedores. Particularmente, los objetivos de este trabajo son, por una parte, brindar a la comunidad de ingenieros clínicos una metodología general que permita la caracterización de las entidades que prestan servicios de mantenimiento y el inventario de los equipos médicos a su cargo y, por otra parte, aplicar técnicas de conglomerados o clustering que permita agrupar a los proveedores de servicios de mantenimiento según la calidad del desempeño de las actividades que realizan.

\section{MÉTODOS}

El estudio se realizó en un Hospital Universitario de III Nivel de complejidad, de la Ciudad de Bogotá D.C., Colombia. Este posee un total de 251 camas, de ellas 209 en hospitalización general, 11 en una única unidad de cuidado intensivo adulto y 31 camas y cunas para atención de la unidad de neonatología. El inventario total de equipos médicos es de 1050 unidades. 
Primero se realizó un estudio de caracterización del inventario. El estudio se llevó a cabo por áreas pilotos y por especialidades de equipos. Se seleccionaron siete áreas pilotos estas fueron: Imágenes Diagnósticas (BB), Unidad Quirúrgica (CC), Unidad de Cuidados Intensivos (DD), Neonatos (FF), Urgencias (AA), Unidad de Cuidados Intermedios (EE), Laboratorio Inmunología (HH), Laboratorio Clínico (GG) y Microbiología (II). Los autores de este trabajo seleccionaron estas áreas debido a la complejidad técnica de los equipos biomédicos y por lo tanto por la mayor probabilidad de que el servicio de mantenimiento sea contratado con un proveedor externo. Las especialidades de equipos tenidas en cuenta fueron: Diagnóstico (A), Tratamiento (B), Monitoreo (C), Apoyo (D) y Laboratorio (E). Estas fueron seleccionadas por ser las que tiene en cuenta el Ministerio de la Protección Social de la República de Colombia (9).

Las variables estudiadas para caracterizar el inventario en las respectivas áreas y especialidades de equipos fueron 12 en total, estas son: cantidad de equipos, razón tiempo de explotación (TE) versus vida útil(VU), valor total de inventario (Valor Inv), por ciento que representa el valor del inventario de los equipos versus el valor del inventario total analizado (\% del Valor Inv Total), costo del servicio del mantenimiento externo, tercerizado o contratado (Costo Mtto Externo), costo del servicio de mantenimiento propio o en casa (Costo Mtto Propio Piezas y Repuestos), la razón costo de mantenimiento propio versus costo del servicio del mantenimiento externo (Costo Propio/Costo Mtto Ext), costo total de mantenimiento, o sea externo más el propio, \% del costo de mantenimiento en relación con el costo total (\% costo total de mtto) y la razón costo del servicio versus costo de adquisición $(\mathrm{CS} / \mathrm{CA})$.

Seguidamente se elaboró una herramienta tipo encuesta con el objetivo de caracterizar a los proveedores de servicios. Las variables recabadas por medio de la encuesta fueron 20 en total (Tabla 1): denominación y registro de la empresa, fecha de inicio del contrato, fecha de finalización del contrato, duración del contrato (Duración en años), Número o cantidad de equipos en el contrato (NEquip), si es un contrato de garantía (Garantía), si el contrato incluye repuestos (REP), número de visitas al año estipuladas en el contrato (NvisitasY), especialidades de los equipos por tipo (Tipoequipos), cantidad de ingenieros y técnicos que tiene la empresa (TotalRHEmpresa), cantidad de ingenieros y técnicos destinados a atender el contrato (TotalRHContrato), experiencia del recurso humano de la empresa en (años, ExpersonalY), distancia a que se encuentra el proveedor del hospital en metros (DistPHUS), cantidad de contratos que maneja la empresa (NContratos), años experiencia de la empresa 
en el negocio (ExpEmp), si ofrece servicios en línea (SevL), si posee stoks de piezas de repuesto en el país (BacUp) y si entrena a usuarios y operadores (EntUsu y EnTec). Estas variables son las más típicas para realizar una caracterización adecuada de los proveedores de servicios de mantenimiento (8).

Se realizó un seguimiento de las variables tiempo de respuesta (RT) (6), duración real de las intervenciones de mantenimiento (DR) (6) de cada de proveedor de servicio de mantenimiento y del servicio en casa que generaron órdenes de trabajo; así como el tiempo de cambio de estado (TAT) (6) y la disponibilidad del equipamiento $(5,6)$, con el objetivo de hacer un seguimiento de la calidad del servicio y obtener posibles niveles de agrupamiento de estas variables y las que caracterizan a las instituciones.

\section{RESULTADOS}

Caracterización de los equipos biomédicos

Se realizó la caracterización del inventario con una población de 264 equipos. Esto representó un 53,4\% del total seleccionada. Estos 264 equipos se seleccionaron teniendo en cuenta de que estuviera el $100 \%$ de la muestra de los posibles proveedores de servicios a encuestar. Esta fase del estudio arrojó los siguientes patrones:

1. El equipamiento puede ser catalogado de "viejo", pues la razón TE/VU tiene un valor promedio de 0,78. Existiendo valores notablemente elevados de TE/ VU tales como es el caso de Neonatos, Microbiología, y Unidad Quirúrgica con valores de, 1,01, 0,92, 1,19 respectivamente

2. El costo total del mantenimiento de los servicios contratados asciende a un valor del 97,6 \% unidades monetarias. En oposición al costo de mantenimiento propio o en casa, que sólo representa el $2,4 \%$ del total del costo del mantenimiento.

3. Las áreas de Imágenes Diagnósticas (BB), Unidad Quirúrgica (CC) y UCI (DD) consumen el 90,3\% del valor total de inventario de los equipos instalados en las 7 áreas analizadas. Coincidentemente consumen el 87,7\% del costo total del mantenimiento estando solo el 50,0 \% de los equipos involucrados en este costo.

4. El valor promedio de la razón $\mathrm{CS} / \mathrm{CA}$ es de 8,61 \% anual. Se puede decir que todos los valores son elevados, muy por encima de lo encontrado en lo reportado en otros estudios $(5,6)$. Es notable destacar que existen valores 
notablemente elevados tales como, UCI (DD), Microbiología (II) y Laboratorio clínico; con valores de $27,8,23,8$ y $21,4 \%$ respectivamente.

Figura 1. Análisis de la variable TAT por proveedor de servicios

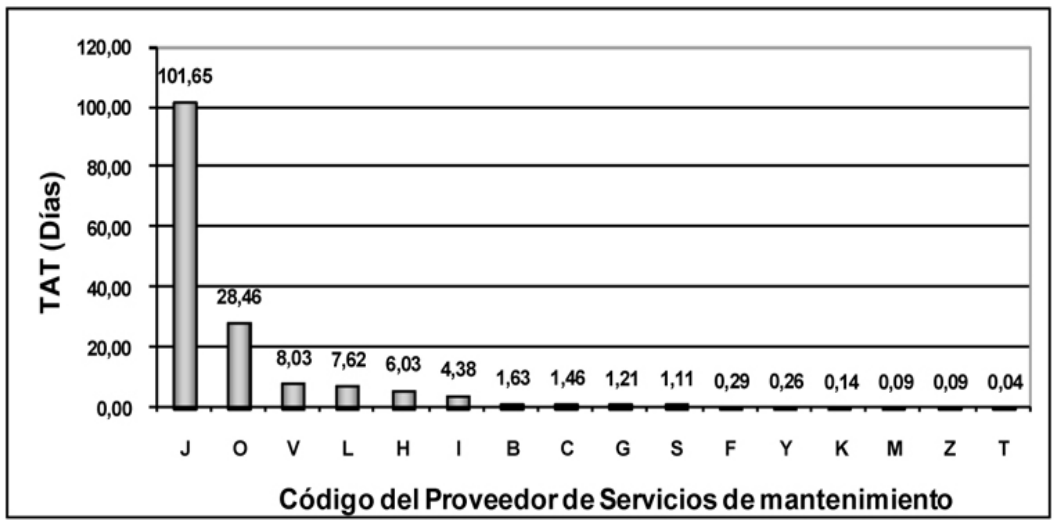

Caracterización de los proveedores de mantenimiento

Se caracterizó el $100 \%$ de la muestra de los proveedores de mantenimiento, esto es una cantidad de 26 proveedores (codificados con las letras del alfabeto desde la A hasta la Z), incluyendo al servicio en casa como un proveedor más. Esta fase arrojó los siguientes patrones:

1. En términos de volumen o cantidad de equipos contratados este representa el $46,7 \%$ de la población de equipos.

2. De este $46,7 \%$ el $62,2 \%$ se encuentra en manos de un proveedor de servicios de mantenimiento externo o tercerizado.

3. Las empresas se encuentran a una distancia cercana a la institución de salud, siendo el valor más común de $10 \mathrm{~km}$ de distancia, siendo la duración de contrato más común de 1 año.

4. Las empresas pueden ser catalogadas con experiencia dentro del gremio, siendo 25 años el valor más repetido; de pequeñas, siendo el valor de 3 personas entre técnicos e ingenieros como total de recurso humano en las mismas. El 15,4 \% de las empresas no posee stock de repuestos en el país. El 11,5\% no ofrece servicios de asistencia en línea. Estas destinan a 3 especialistas, como valor más común, entre técnicos e ingenieros para atender a los equipos bajo contrato de servicios. 
Estudio del agrupamiento de los proveedores de servicios

La Figura 2 muestra el ordenamiento de los proveedores de servicios según la variable TAT (en días). Observe como los valores de TAT que ostentan los proveedores se dividen en tres grandes grupos: Valores elevados (TAT $>6$ días), incluso con valores no permisibles en el orden de 101 días, o sea más de tres meses (proveedor J, O, V, L, y H), Valores intermedios $(1<\mathrm{TAT}<6)$, o sea menores a una semana (proveedores I, B, C, G y S) y Valores bajos $(\mathrm{TAT}<1$ ), o sea menores a un día, lo cual es un valor de TAT realmente bueno (proveedores F, Y, K, M, Z, y T).

Un análisis integral de las características de los proveedores se puede hacer realizando un estudio de agrupamiento. Este nos permite agrupar a los proveedores en conglomerados permitiendo identificar cuales son sus características fundamentales. Se obtuvieron 4 conglomerados o grupos. Pudiendo calificar a los proveedores de servicios de la siguiente manera:

1. Grupos ( 1 y 3): Mejor desempeño, menores valores de TAT, RT y DR; cuyos proveedores son: O, L, C, B, I, S, H, F, G; representan el $56 \%$ del total; con valores de TAT entre: 1,4 días $<$ TAT $<2,56$ días como promedio.

2. Grupo (0): Desempeño medio, cuyos proveedores son: V, M, K, Z, T, Y; representan el $38 \%$; con valores de TAT iguales a 9,79 días como promedio. 3. Grupo (2): con bajos desempeños, por poseer valores altos de TAT (101 días como promedio); con único proveedor: J, representa el $6 \%$ del total de la muestra analizada.

Figura 2. Conglomerados o clústeres obtenidos por proveedor de servicios en función de la variable TAT en horas

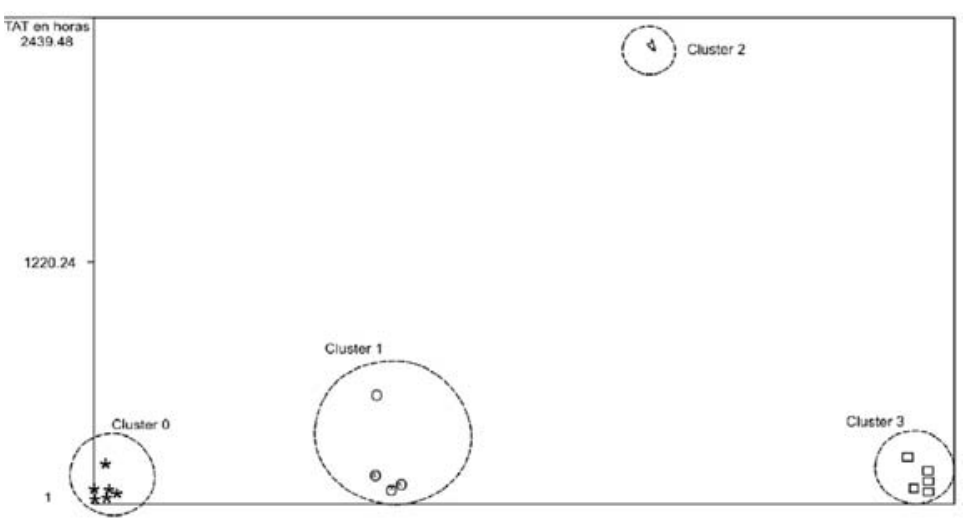




\section{DISCUSIÓN}

La disponibilidad de los equipos se estudió como una variable más dentro del seguimiento de la calidad del servicio. Se obtuvieron valores muy buenos de esta variable (por encima de $90 \%$ para tres de los cuatro grupos de proveedores obtenidos). Sin embargo, este valor es debido a la cantidad de horas tan elevada que los equipos tienen que operar para cumplir la función misional dentro de los servicios de salud (alcanzando valores de 10240,05 horas para el periodo analizado). Se puede decir que los valores deficientes de TAT son "enmascarados" por lo buenos resultados de la disponibilidad.

Tabla 1. Clústeres obtenidos

\begin{tabular}{|c|c|c|c|c|}
\hline \multirow{2}{*}{ Atributos } & \multicolumn{4}{|c|}{ Clústeres } \\
\hline & 0 & 1 & 2 & 3 \\
\hline Proveedores & $\begin{array}{l}\mathrm{V}, \mathrm{M}, \mathrm{K}, \\
\mathrm{Z}, \mathrm{T}, \mathrm{Y}\end{array}$ & $\mathrm{O}, \mathrm{I}, \mathrm{C}, \mathrm{B}$ & $\mathrm{J}$ & $\begin{array}{c}\text { I, S, H, } \\
\text { F, G }\end{array}$ \\
\hline Cantidad y \% & $6(38 \%)$ & $4(25 \%)$ & $1(6 \%)$ & $5(31 \%)$ \\
\hline Duración (años) & 1,11 & 0,92 & 5,00 & 1,68 \\
\hline Nequip & 1,32 & 41,49 & 266,00 & 4,75 \\
\hline NvisitasY & 4,86 & 3,25 & 2,00 & 3,59 \\
\hline TotaIRHEmpresa & 10,96 & 5,75 & 3,00 & 20,09 \\
\hline TotalRHContrato & 3,68 & 3,75 & 3,00 & 3,00 \\
\hline TotalRHEqCont & 3,19 & 0,26 & 0,01 & 1,15 \\
\hline ExpPersonalY & 12,72 & 8,00 & 8,00 & 7,82 \\
\hline DistKm & 11,02 & 3,32 & 0,00 & 10,70 \\
\hline Ncontratos & 96,17 & 10,00 & 0,00 & 94,03 \\
\hline ExpEmp & 34,82 & 7,87 & 7,50 & 20,29 \\
\hline \multicolumn{5}{|l|}{ SerL } \\
\hline SI & 5,91 & 5,00 & 2,00 & 5,09 \\
\hline NO & & 1,00 & 1,00 & 2,00 \\
\hline \multicolumn{5}{|l|}{ Backup } \\
\hline SI & 0,00 & 5,00 & 2,00 & 5,00 \\
\hline NO & 4,91 & 1,00 & 1,00 & 2,09 \\
\hline \multicolumn{5}{|l|}{ RepP } \\
\hline SI & 6,91 & 5,00 & 2,00 & 4,09 \\
\hline NO & 1,00 & 1,00 & 1,00 & 3,00 \\
\hline \multicolumn{5}{|l|}{ EnUsu } \\
\hline NO & 1,00 & 4,00 & 1,00 & 1,00 \\
\hline SI & 6,91 & 2,00 & 2,00 & 6,09 \\
\hline \multicolumn{5}{|l|}{ EnTec } \\
\hline SI & 3,91 & 2,00 & 2,00 & 6,09 \\
\hline NO & 4,00 & 3,00 & 1,00 & 1,00 \\
\hline RT (horas) & 32,98 & 49,72 & 43,33 & 54,46 \\
\hline DR (horas) & 2,08 & 185,25 & 2396,15 & 6,97 \\
\hline TAT (horas) & 35,06 & 234,99 & 2439,48 & 61,44 \\
\hline Disponibilidad (\%) & 87,0 & 98,0 & 98,0 & 93,0 \\
\hline
\end{tabular}


Desafortunadamente el proveedor J es el peor proveedor de servicios, este es el servicio de mantenimiento en casa; o sea el Dpto. de Ingeniería Clínica del hospital. Las posibles razones que influencian estos resultados pueden estar asociadas al alto índice de obsolescencia de los equipos, por consiguiente el gran número de fallas y a la falta de entrenamiento y cuidado en la operación de los equipos. Nuevas estrategias deben presentarse para mejorar la calidad del servicio Estas pueden ir desde aumentar el número de especialistas y técnicos dentro del Dpto., hasta mejorar los niveles de entrenamientos a usuarios y técnicos; y aplicar adecuadas técnicas de priorización del equipamiento. Se recomienda hacer estudios posteriores del número de fallas y la cantidad de órdenes de servicio de mantenimiento preventivo en función de la cantidad de equipos y el número de especialistas de cada proveedor de servicios, incluido el servicio en casa.

De este estudio se puede concluir que:

1. Los proveedores de servicios con peores desempeños son los que tienen más equipos bajo su cargo, adicionalmente estos son los equipos de mayor complejidad tecnológica (diagnóstico)

2. Los proveedores de servicios con peores desempeños son los que más distantes se encuentran del hospital.

3. Las técnicas de minería de datos constituyen de manera efectiva a evaluar el desempeño de los proveedores de servicios de mantenimiento

De este trabajo se desencadenan oportunidades de mejoras. En primer lugar, se debe continuar realizando el seguimiento de la variable TAT, a través de las órdenes de trabajo, con el objetivo de aplicar una técnica predictiva para obtener valores del TAT en función de las características de las empresas proveedoras de servicios de mantenimiento y las variables RT y DT. En segundo lugar, se debe continuar con el seguimiento para incorporar al estudio a aquellos proveedores de servicios de mantenimiento que no han reportado órdenes de servicios; debido o bien porque los equipos bajo su cargo no han fallado, o bien por no existir programaciones de mantenimiento en el período a analizado *

Agradecimientos: A COLCIENCIAS por los recursos brindados en la convocatoria 459/08 para la financiación del estudio. Al Hospital Universitario la Samaritana por su apoyo y colaboración en la realización de esta investigación. 


\section{REFERENCIAS}

1. American college of clinical Engineering (ACCE) [Internet]. Disponible en: http:// www.accenet.org/default.asp?page=about\&section=definition. Consultado Junio de 2009.

2. Antunez E., Do Vale M., Mordelet P., y Grabois V. Gestão da Tecnologia Biomédica. Tecnovigilância e Engenharia Clínica, Ediciones científicas ACODESS; 2002: 210.

3. Ministerio de la Protección Social. Modelo de Evaluación y Gestión del Equipamiento Biomédico [Internet]. Disponible en: http://www.minproteccionsocial.gov.co/VBeContent/Library/ documents/DocNewsNo14710DocumentNo5095.pdf. Consultado Junio de 2009.

4. Duarte G, Sackett $P$, Evans S. Migration and outsourcing. IEEE Trans Manufacturing Engineer. 2004; 3(4): 44-48.

5. Cohen T. Benchmark indicators for medical equipment repair and maintenance. Biomed Instrum Technol.1995; 29(4); 308-320.

6. Cohen T. Validating medical equipment repairs and maintenance metrics: Parts II. Biomed Instrum Technol.1998; 32(2): 136-144.

7. Segupta K, Zviran M, Measuring user satisfaction in an outsourcing environment. IEEE Trans on engineering management. 1997; 44(4): 414-421.

8. ECRI. In House servicing of X-ray and CT Equipment, Health Technology Management; Winter 1993; 33(1): 312-318.

9. INVIMA. Decreto 4725 de 2005 [Internet]. Disponible en http://www.minproteccionsocial.gov.co/ VBeContent/library/documents/DocNewsNo15472DocumentNo2802.PDF . Consultado Junio 2009.

10. WEKA v 3.6. [Internet]. Disponible en: http://www.cs.waikato.ac.nz/ml/weka/ index_downloading.html. Consultado junio de 2009. 